\title{
Development of clinical decision rules to predict recurrent shock in dengue
}

\author{
Nguyen Tien Huy ${ }^{1,2+}$, Nguyen Thanh Hong Thao ${ }^{3 \dagger}$, Tran Thi Ngoc Ha ${ }^{4}$, Nguyen Thi Phuong Lan ${ }^{5}$,

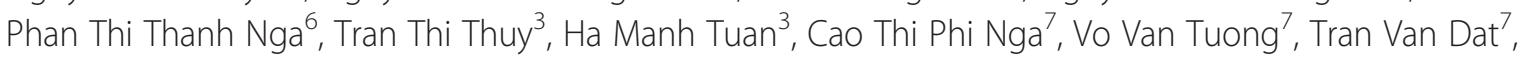 \\ Vu Thi Que Huong ${ }^{4}$, Juntra Karbwang ${ }^{2}$ and Kenji Hirayama ${ }^{1 *}$
}

\begin{abstract}
Introduction: Mortality from dengue infection is mostly due to shock. Among dengue patients with shock, approximately $30 \%$ have recurrent shock that requires a treatment change. Here, we report development of a clinical rule for use during a patient's first shock episode to predict a recurrent shock episode.

Methods: The study was conducted in Center for Preventive Medicine in Vinh Long province and the Children's Hospital No. 2 in Ho Chi Minh City, Vietnam. We included 444 dengue patients with shock, 126 of whom had recurrent shock (28\%). Univariate and multivariate analyses and a preprocessing method were used to evaluate and select 14 clinical and laboratory signs recorded at shock onset. Five variables (admission day, purpura/ecchymosis, ascites/pleural effusion, blood platelet count and pulse pressure) were finally trained and validated by a 10-fold validation strategy with 10 times of repetition, using a logistic regression model.

Results: The results showed that shorter admission day (fewer days prior to admission), purpura/ecchymosis, ascites/pleural effusion, low platelet count and narrow pulse pressure were independently associated with recurrent shock. Our logistic prediction model was capable of predicting recurrent shock when compared to the null method $(P<0.05)$ and was not outperformed by other prediction models. Our final scoring rule provided relatively good accuracy (AUC, 0.73; sensitivity and specificity, 68\%). Score points derived from the logistic prediction model revealed identical accuracy with AUCs at 0.73 . Using a cutoff value greater than -154.5 , our simple scoring rule showed a sensitivity of $68.3 \%$ and a specificity of $68.2 \%$.
\end{abstract}

Conclusions: Our simple clinical rule is not to replace clinical judgment, but to help clinicians predict recurrent shock during a patient's first dengue shock episode.

\section{Introduction}

Dengue virus infection has become an important global problem. It is quickly spreading to South America and Africa [1]. It is estimated that 20,000 patients die from this disease each year from among the 2.5 billion people who are at risk for dengue in over 100 countries [2]. The pathogenesis of dengue infection is not completely understood. Several mechanisms have been proposed, including a virulence factor [3-5], secondary infection [5], host genetic factors [6,7], host immune response [8-10], memory T-cell-mediated pathogenesis [8], suppressed Th1 and/or

\footnotetext{
* Correspondence: hiraken@nagasaki-u.ac.jp

${ }^{\dagger}$ Equal contributors

'Department of Immunogenetics, Institute of Tropical Medicine (NEKKEN),

Nagasaki University, 1-12-4 Sakamoto, Nagasaki 852-8523, Japan

Full list of author information is available at the end of the article
}

predominant Th2 responses [9], cytokine tsunami [10], anti-nonstructural 1 protein antibodies that cross-react with vascular endothelium [11] and host physiological factors [12].

Dengue disease, as defined according to the 1997 World Health Organization (WHO) classifications, ranges from asymptomatic or dengue fever to severe dengue hemorrhagic fever and dengue shock syndrome (DSS) [13]. Recently, in the 2009 revised dengue classification system proposed by Dengue Control, the WHO has added a classification of the severity of the disease according to the presence of dengue warning signs [14].

To date, there is no approved vaccine or antiviral drug to treat for this disease. Vector control, early appropriate treatment and educational programs are currently the only ways to reduce mortality and the global disease

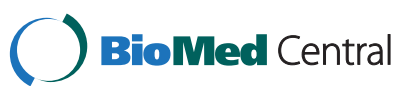


burden [14-17]. Mortality can reach $10 \%$ to $20 \%$ without early appropriate treatment [18]. Death due to dengue infection is reportedly 50 times higher in dengue patients with shock than in those without shock [19]. Among shock patients, approximately 30\% have recurrent shock that reportedly affects the therapy protocol [20]. To the best of our knowledge, however, no previous report has described a tool for prediction of patients who will develop a second dengue shock episode. In this study, we developed a model to predict, at the time of the first shock episode, patients who would develop recurrent shock.

\section{Materials and methods Study design}

The current study was performed at the Center for Preventive Medicine in Vinh Long province and the Children's Hospital No. 2 in Ho Chi Minh City, Vietnam. The study design was a prospective cohort analysis of the clinical signs and laboratory test values of children (ages 6 months to 15 years) that were recorded around the time of the first shock episode. The entry criteria were suspected clinical dengue infection with proven dengue virus on the basis of laboratory evidence and hypovolemic shock that occurred between January 2002 and December 2007. Proven dengue virus infection was diagnosed if the virus was isolated, if RNA was detected by real-time PCR assay or if the serological assay was positive, as fully described in our previous publications $[7,21,22]$. DSS was classified according to the WHO 1997 classification criteria [13] without fulfilling the criterion for presence of thrombocytopenia [23]. Exclusion criteria were the presence of chronic illness or massive bleeding that required blood transfusion.

Around the time of shock, the relevant patient history regarding clinical symptoms and signs, as well as the laboratory parameters listed in Table 1 , were recorded. These variables are widely used in our hospitals for the diagnosis and monitoring of dengue shock patients. Pleural effusion and ascites were detected by chest X-ray and ultrasound. A patient was defined as having developed recurrent shock if he or she had received adequate fluid according to the WHO 1997 guidelines for volume replacement [13] and had tachycardia, abnormal coolness of limbs and a pulse pressure $\leq 25 \mathrm{mmHg}$ that had previously reached a level $\geq 30 \mathrm{mmHg}$ [20].

The minimal required sample size was determined by rule of thumb, in which at least ten patients per group were required for each included predictive variable $[24,25]$. We aimed to use less than ten variables to build prediction models for ease of use in clinical practice. The required sample size was at least 100 participants per group. This study was approved by the institutional ethical review committees of the Institute of Tropical Medicine, Nagasaki University, and the Pasteur Institute in Ho Chi Minh City. Written informed consent from all participants' parents or guardians was required upon enrollment.

\section{Univariate and multivariate analyses}

The primary outcome variable was recurrent shock diagnosis at the time of hospital discharge. Univariate

Table 1 Clinical characteristics, laboratory parameters and univariate analysis ${ }^{\mathrm{a}}$

\begin{tabular}{|c|c|c|c|c|}
\hline Characteristics & $\begin{array}{l}\text { Single shock } \\
(N=318)\end{array}$ & $\begin{array}{l}\text { Recurrent shock } \\
(N=126)\end{array}$ & Missing data (\%) & $P$-value \\
\hline Age (years) & $10(0.25 \text { to } 15)^{b}$ & $10(0.5$ to 15$)$ & 0 & 0.95 \\
\hline Females/total & $171 / 318$ & $73 / 126$ & 0 & 0.43 \\
\hline Admission day & $3.97 \pm 1.20^{c}$ & $3.71 \pm 1.08$ & $1(0.2)$ & 0.035 \\
\hline Days of shock & $4.95 \pm 1.34$ & $4.67 \pm 1.38$ & $14(3.2)$ & 0.054 \\
\hline Petechia & $144 / 318(45 \%)$ & $61 / 126(48 \%)$ & 0 & 0.55 \\
\hline Tourniquet test & $12 / 318(4 \%)$ & $5 / 126(4 \%)$ & $2(0.5)$ & 0.93 \\
\hline Nose/gum bleeding & 40/318 (13\%) & $21 / 126(17 \%)$ & 0 & 0.26 \\
\hline Purpura/ecchymosis & 98/318 (31\%) & $62 / 126(49 \%)$ & 0 & $<0.001$ \\
\hline Gl bleeding & $36 / 318(11 \%)$ & 23/126 (18\%) & 0 & 0.052 \\
\hline Ascites/pleural effusion & $114 / 318(36 \%)$ & $92 / 126(73 \%)$ & $1(0.2)$ & $<0.001$ \\
\hline HCT (\%) & $43.01 \pm 5.83$ & $43.50 \pm 5.50$ & $3(0.7)$ & 0.43 \\
\hline $\operatorname{PLTs}\left(\times 10^{3} / \mu \mathrm{l}\right)$ & $110.59 \pm 56.10$ & $96.63 \pm 60.34$ & $16(3.6)$ & 0.024 \\
\hline WBC count $\left(\times 10^{3} / \mu l\right)$ & $5.55 \pm 3.75$ & $4.98 \pm 2.73$ & $44(9.9)$ & 0.141 \\
\hline Pulse pressure $(\mathrm{mmHg})$ & $18.41 \pm 6.26$ & $16.31 \pm 7.24$ & 0 & 0.001 \\
\hline
\end{tabular}

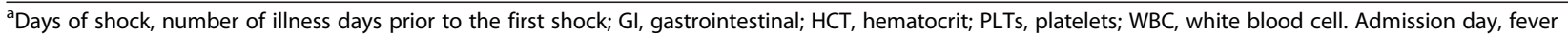
days prior to admission and day 1 of illness were assigned as the day of fever onset. ${ }^{b}$ Median (minimum, maximum) calculated using the Mann-Whitney $U$ test for continuous variables not normally distributed. 'Mean \pm SD calculated using Student's $t$-test for continuous variables normally distributed. 
analysis was performed using SPSS software version 16.0 (SPSS, Inc, Chicago, IL, USA). Measures of skewness and kurtosis were used to test the normal distribution of continuous variables. Student's $t$-test was used for continuous variables normally distributed, and the MannWhitney $U$ test was used for continuous variables that were not normally distributed. $X^{2}$ analysis was used for categorical variables. A difference with a $P$ value $<0.05$ was considered significant. Multivariate logistic regression analysis was used to find the independent predictors of recurrent shock using MedCalc version 11.0 statistical software (MedCalc Software, Ostend, Belgium).

\section{Missing data analysis}

Missing data ranged from $0 \%$ to $9.9 \%$. Little's missing completely at random test [26] was performed using SPSS software version 16.0, which showed a nonsignificant result $(P>0.10)$, suggesting that the data were missing completely at random. Therefore, multiple imputation algorithms were used to analyze the missing data using NORM software version 2.03 [27]. The multiple logistic regression model was employed to input missing data.

\section{Preprocessing variable selection}

To increase the performance of the prediction model [28], a preprocessing method was employed to reduce the number of variables using Weka 3.7.7 software [29]. We used the assessment method in WrapperSubsetEval [30] combined with the best first search method in the forward direction [31] and the learning scheme of our logistic regression prediction model (Logistic). A tenfold cross-validation method was used with the number of folds set at five and the seed set at one.

\section{Training and validation of the prediction model}

Because a simpler rule would be preferred by clinicians, particularly in remote areas, we built a simple traditional rule using a logistic regression model [32]. Logistic was built and compared to ZeroR, a baseline model without predictive power, to identify the predictive power of the Logistic model. The overall performance of the datamining model was assessed by calculation of the area under the curve (AUC) from the receiver operating characteristic (ROC) curve [33]. Weka 3.7.7 software [29] was used to train and validate all models in a tenfold validation strategy with ten rounds of ten repetitions as previously described $[34,35]$. Briefly, the whole data set was randomly split into ten equal subsets (Figure 1). For ten times, nine subsets were used to train the model and the remaining subset was used to validate the AUC of each model. The cross-validation was repeated 10 times to yield a total of 100 AUC values for each model. The overall accuracy of these models, represented by the mean and standard deviation of 100 AUC values, was compared using the corrected, resampled $t$-test [36]. Differences between models with $P$ values $<0.05$ were considered significant.

Initially, we used the default settings of the Weka software to build the prediction model. Next, we optimized parameters to calculate the highest AUC value. The full description of parameter-setting is presented in Additional file 1.

Score points were derived by multiplying and rounding regression coefficients to calculate the lowest integer, then simplifying them to achieve a scoring system. The optimal cutoff value was chosen as the $q$ value of the ROC curve, at which sensitivity equals specificity.

\section{Comparison of logistic regression prediction model with the other ten prediction models}

Ten binary prediction models (artificial neural network (ANN), $k$-nearest neighbors algorithm $(k N N), C 4.5$ decision tree (J48), LogitBoost classification algorithm (LgBoost), logistic regression tree (LRT), naïve Bayes classifier (NaiveB), random forests (RF), random subspace (RSSpace), sequential minimal optimization (SMO) for support vector machines and a combination of ANN, LgBoost and RSSpace (Vote)) were built as described above. The overall accuracy of these rules was compared using the mean and standard deviation of 100 AUC values per model by using the corrected, resampled $t$-test [36].

\section{Results}

\section{Patient characteristics and univariate analysis}

A total of 444 proven dengue patients with shock, including 318 patients (72\%) with a single shock episode and $126(28 \%)$ with recurrent shock, were enrolled (Table 1). No deaths were recorded in the study. There were no significant differences in age and gender between the control (single shock) and recurrent shock DSS groups. The mean admission day (mean number of illness days prior to admission, and day 1 of illness was assigned as the day of fever onset) for the recurrent shock group was significantly lower than that of the single shock group $(P<0.05)$. The mean of the day of shock (illness days prior to the first shock) was slightly lower in the recurrent shock group than in the single shock group $(P=0.054)$. For the clinical and laboratory parameters, purpura/ecchymosis $(P<0.001)$, ascites/pleural effusion $(P<0.001)$ and lower platelet count $(P<0.05)$ and narrow pulse pressure $(P<0.001)$ were found more commonly in the recurrent shock group than in the single shock group. Gastrointestinal bleeding was closely associated with recurrent shock $(P=0.052)$. No significant differences between the two groups were found for petechia, tourniquet test, nose/gum bleeding, hematocrit and white blood cell count. 


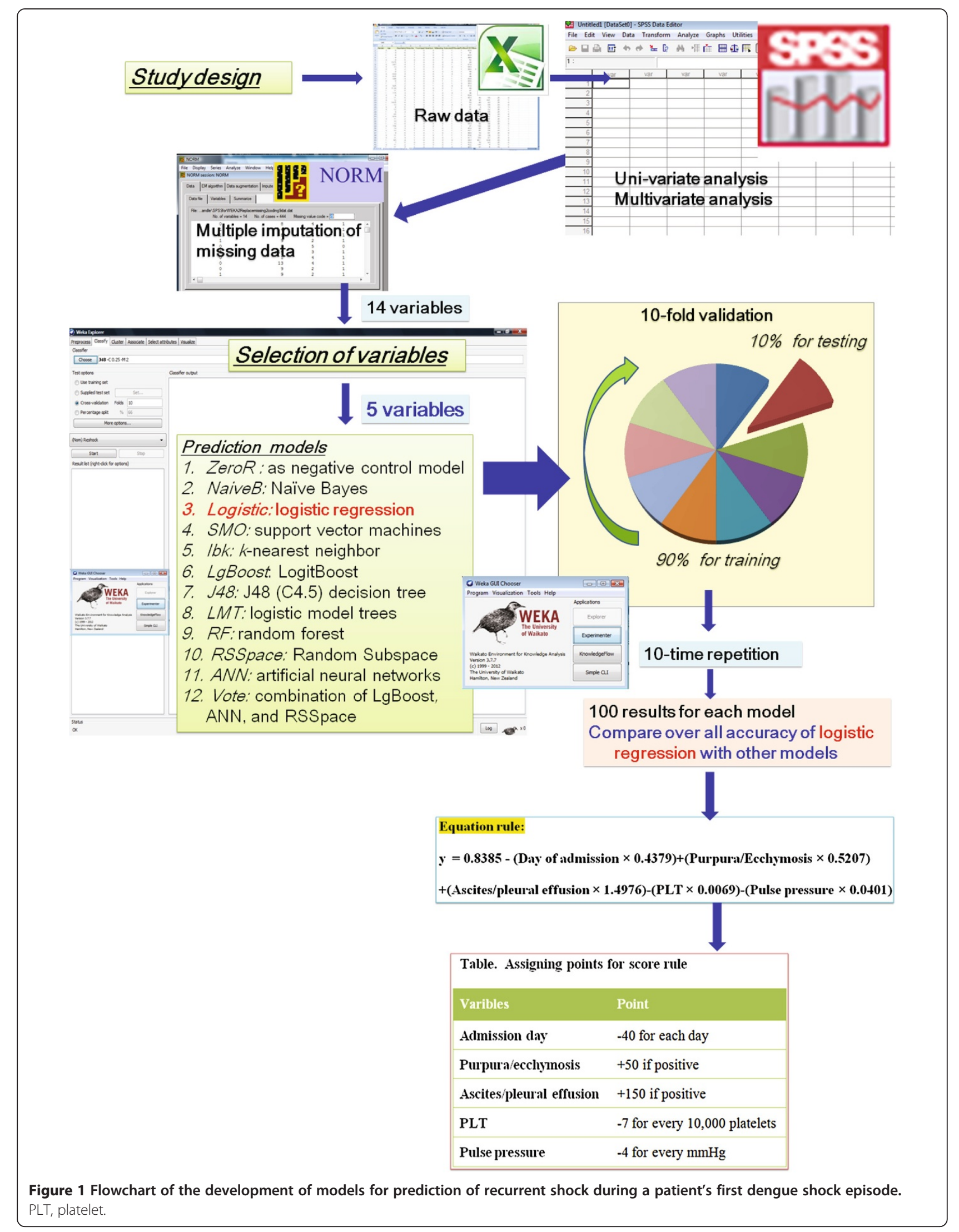




\section{Multivariate analysis and development of clinical decision rule}

Because the clinical rule should be reliable and easy to apply [32], we aimed to develop a simple rule to predict the recurrent shock patients using the logistic regression model. After the preprocessing method of variables reduction, nine variables, including age, admission day, day of shock, petechia, purpura/ecchymosis, gastrointestinal bleeding, ascites/pleural effusion, platelet count, and pulse pressure, remained and were analyzed using a multivariate logistic regression model. The results in Table 2 show that admission day, purpura/ecchymosis, ascites/pleural effusion, blood platelet count, and pulse pressure, independently correlated with recurrent shock in a multivariate model $(P<0.05)$.

Five variables (admission day, purpura/ecchymosis, ascites/pleural effusion, blood platelet count and pulse pressure) were further trained and validated using a tenfold validation strategy with ten rounds of repetition [34,35] using Weka 3.7.7 software [29]. Next, scores were derived by multiplying and rounding regression coefficients to calculate the lowest integer, then simplifying the coefficients to achieve a scoring system (Table 3). The total score for patients in the whole data set ranged from -406 points to +53 points. Figure 2 shows the performance of the logistic models built by using the equation and the scoring systems, revealing identical accuracy with AUCs at 0.73 when the whole data set was applied. Using a cutoff value greater than -154.5 , the simple point rule revealed a sensitivity of $68.3 \%$ and a specificity of $68.2 \%$.

\section{Comparison of 11 prediction models}

In this study, 11 popular data-mining methods (ANN, $k \mathrm{NN}$, J48, LgBoost, LRT, Logistic, NaiveB, RF, RSSpace, SMO and Vote) were trained and compared to the baseline ZeroR model to identify the predictability power of the built models. Five variables (admission day, purpura/ ecchymosis, ascites/pleural effusion, blood platelet count and pulse pressure) were computed using a ten-fold validation strategy. The results showed that all 11 prediction models possessed AUC values between 0.645 and 0.730 (Additional file 2). Compared to the baseline ZeroR method, the 11 prediction models all showed an ability to predict recurrent shock $(P<0.05)$. Among these models, the Vote model demonstrated the highest AUC at 0.730 , followed by LgBoost (0.720), NaiveB (0.705), Logistic (0.703), LRT (0.703), $k \mathrm{NN}(0.696)$, SMO (0.695), ANN (0.695), RF (0.694), RSSpace (0.694) and J48 (0.645). Compared with the other four best models (Vote, LgBoost, NaiveB and Logistic), the J48 model was significantly outperformed in overall accuracy.

\section{Discussion}

Medical doctors often initiate fluid resuscitation by using normal saline solution or Ringer's lactate solution. Most children with DSS recover from shock with this treatment regimen; however, $28 \%$ to $30 \%$ have recurrent shock that requires administration of colloidal solutions and more intensive care [20] (Table 1). Therefore, a clinical decision rule is needed to predict recurrent shock during a patient's first dengue shock episode. In this study, we defined a simple, practical, relatively accurate clinical decision rule (sensitivity and specificity of $68 \%$ ) that can be applied at the bedside [32] to predict recurrent shock in children hospitalized for a first dengue shock episode. The rule contains a list of items with a detailed scoring system and does not require complicated mathematical computation.

Our univariate analysis revealed many factors associated with recurrent shock. There were more signs of purpura/ecchymosis, gastrointestinal bleeding, ascites/ pleural effusion, low platelet count and narrow pulse pressure in recurrent shock patients compared with

Table 2 Multivariate logistic regression model used to predict dengue shock syndrome and nonshock cases ${ }^{\mathrm{a}}$

\begin{tabular}{|c|c|c|c|}
\hline Predictors & OR $(95 \% \mathrm{Cl})$ & Adjusted OR (95\% CI) & $P$-value \\
\hline Age & 1.01 (0.95 to 1.08) & 1.05 (0.98 to 1.13$)$ & 0.1756 \\
\hline Admission day $y^{b, c}$ & 0.82 (0.69 to 0.99$)$ & 0.66 (0.52 to 0.83 ) & 0.0041 \\
\hline Day of shock & 0.91 (0.77 to 1.07$)$ & 0.91 (0.76 to 1.08 ) & 0.3476 \\
\hline Petechia & $1.13(0.75$ to 1.71$)$ & 0.78 (0.46 to 1.30$)$ & 0.3381 \\
\hline Purpura/ecchymosis ${ }^{c}$ & 2.17 (1.43 to 3.32$)$ & 1.78 (1.11 to 2.86$)$ & 0.017 \\
\hline Gl bleeding & 1.75 (0.99 to 3.09) & 1.37 (0.73 to 2.58$)$ & 0.1290 \\
\hline Ascites/pleural effusion ${ }^{c}$ & 0.23 (0.14 to 0.42$)$ & 0.24 (0.13 to 0.43 ) & 0.0001 \\
\hline $\operatorname{PLT}\left(\times 10^{3} / \mu \mathrm{l}\right)^{\mathrm{c,d}}$ & 0.99 (0.99 to 0.99$)$ & 0.99 (0.99 to 0.99 ) & 0.0148 \\
\hline Pulse pressure $(\mathrm{mmHg})^{c, d}$ & 0.96 (0.93 to 0.98$)$ & 0.96 (0.93 to 0.99$)$ & 0.0163 \\
\hline
\end{tabular}

${ }^{\mathrm{a}} \mathrm{Cl}$, confidence interval; $\mathrm{Gl}$, gastrointestinal; OR, odds ratio; PLT, platelet. Dengue shock syndrome refers to dengue hemorrhagic fever, and nonshock cases refers to dengue fever.

${ }^{b}$ OR represents the decremental odds of recurrent shock for every unit decrease of one day in admission days. ${ }^{c}$ Variables were selected for developing the clinical rules. ${ }^{\mathrm{d}} \mathrm{OR}$ represents the incremental odds of recurrent shock for every unit increase of 1,000 platelets per microliter or every $1 \mathrm{mmHg}$ increase in pulse pressure. 
Table 3 Assigning score values for clinical decision rule ${ }^{a}$

\begin{tabular}{ll}
\hline Variables & Points $^{\mathbf{b}}$ \\
\hline Admission day & -40 for each day \\
Purpura/ecchymosis & +50 if positive \\
Ascites/pleural effusion & +150 if positive \\
PLT & -7 for every 10,000 platelets \\
Pulse pressure & -4 for every $1 \mathrm{mmHg}$
\end{tabular}

${ }^{\mathrm{a}} \mathrm{PLT}$, platelet. ${ }^{\mathrm{b}}$ Derived from the following equation: $y=0.8385-$ (day of admission $\times 0.4379)+($ purpura/ecchymosis $\times 0.5207)+($ ascites/pleural effusion $\times 1.4976)-($ PLT $\times 0.0069)-($ pulse pressure $\times 0.0401)$.

patients who had a single shock, similarly to the severity signs of dengue infection [2]. A short duration of fever prior to admission was associated with recurrent shock, suggesting rapid progression of the disease in recurrent shock patients. The mechanism of this phenomenon is unknown, thus further studies are required to clarify it.

Several other data-mining techniques, such as ANN, $k N N$, J48, LgBoost, LRT, NaiveB, RF, RSSpace, SMO and Vote, recently have been developed and used to help the clinician make decisions [37-42]. Though the traditional Logistic model has been used extensively to develop decision rules, other modern techniques are underutilized in clinical practices. It has been proposed that intensive computer-based data-mining classifiers outperform the traditional classification methods in several data sets [43-47]. However, this superiority has not been obvious in other data sets $[48,49]$. Thus, it is important to compare several models in order to find an optimal clinical decision-making model for a particular prediction [50].

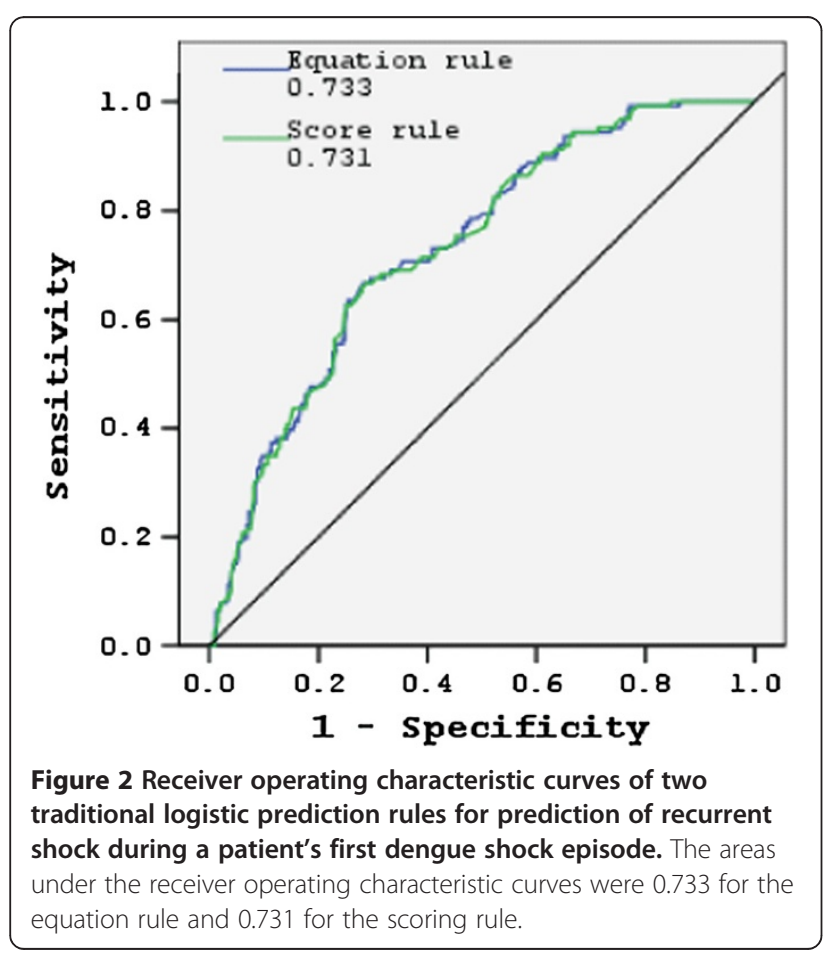

In our present study, we simultaneously developed, tested and compared the traditional Logistic model with ten common prediction models for critical care in dengue infection. Our results show that all 11 models had significant power to predict, around the time of first dengue shock episode, which patients would develop a second shock episode. Five models (Vote, LgBoost, NaiveB, Logistic and LRT) had AUCs >0.7, which is considered an acceptable discrimination level [51]. Among these five models, no prediction rule had superior overall accuracy compared to the other rules. Furthermore, no prediction models were significantly better than the relatively simple logistic regression model in terms of overall accuracy (AUC).

There are several limitations of this study. First, we analyzed data from only two hospitals in southern Vietnam. Therefore, the results would have been different if we had used data from other hospitals, particularly those in other countries, where the clinical characteristics, epidemiology and outcomes of dengue infection are different. Second, the overall accuracy of the final model was not so high, thus more markers are needed to improve the efficacy of the rule in future. Third, we did not include treatment variables at the time of first dengue shock episode (such as initial fluids and length of stay in the ICU), Pediatric Risk of Mortality or Sequential Organ Failure Assessment score variables (such as heart rate, respiratory rate and creatinine level), which might have improved the accuracy of the prediction rule. In addition, we used only one software package to build all prediction models. Other software could produce different results regarding the best model. Thus, further prospective studies using data from different regions are required for external validation of the results of this study.

\section{Conclusions}

Recurrent shock occurs in about $28 \%$ of dengue-infected patients with shock. We derived a simple clinical decision rule with a sensitivity and specificity of $68 \%$, which could help clinicians treating dengue patients during the early stage of a first shock episode to predict the development of recurrent shock. The usefulness of this decision rule needs to be validated by several independent studies in the future.

\section{Key messages}

- Several prediction models were capable of predicting reshock in children who had a first dengue shock (AUC >0.7).

- The simple traditional logistic regression model derived from five factors (admission day, purpura/ ecchymosis, ascites/pleural effusion, blood platelet count and pulse pressure) provided relatively good accuracy with an AUC of 0.73 . 


\section{Additional files}

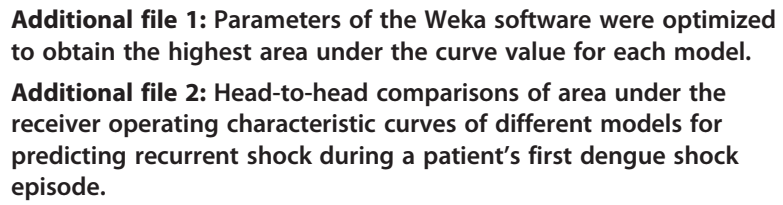

\section{Abbreviations}

ANN: Artificial neural network; AUC: Area under the curve; DF: Dengue fever; DHF: Dengue hemorrhagic fever; DSS: Dengue shock syndrome; J48: C4.5 decision tree; kNN: k-nearest neighbor; LgBoost: LogitBoost; LRT: Logistic regression tree; NaiveB: naïve Bayes classifier; RF: Random forests; RSSpace: Random subspace, ROC, receiver operating characteristic; SMO: Sequential minimal optimization.

\section{Competing interests}

The authors have no competing interests with commercial or other affiliations.

\section{Authors' contributions}

NTH and KH conceived and planned the study. NTH, NTHT, TTNH, NTPL, PTTN, TTT, HMT, CTPN, WT, TVD, VTQH, JK and KH carried out the study. NTH, NTHT, TTNH, NTPL, VTQH, JK and KH analyzed the data and wrote the paper. NTH, NTHT, TTNH, NTPL, TTT, HMT, CTPN, WT, TVD, VTQH and KH contributed reagents and materials. All authors read and approved the final manuscript.

\section{Acknowledgments}

We thank all the members of the Laboratory of Arbovirus, Ho Chi Minh City, Vietnam. We also express our appreciation to the staff of Children's Hospital No. 2 in Ho Chi Minh City and the Center for Preventive Medicine in Vinh Long Province of the Mekong Delta. This study was supported in part by the Global Centers of Excellence Program (2008-2012) and the Japan Initiative for Global Research Network on Infectious Diseases (J-GRID) (to KH). This work was also supported in part by a grant-in-aid for scientific research from Nagasaki University (2007-2009) (to NTH). The funders had no role in the study design, data collection and analysis, decision to publish or preparation of the manuscript.

\section{Author details}

${ }^{1}$ Department of Immunogenetics, Institute of Tropical Medicine (NEKKEN), Nagasaki University, 1-12-4 Sakamoto, Nagasaki 852-8523, Japan. ${ }^{2}$ Department of Clinical Product Development, Institute of Tropical Medicine (NEKKEN), Nagasaki University, 1-12-4 Sakamoto, Nagasaki 852-8523, Japan. ${ }^{3}$ Children's Hospital, No. 2, 14 Ly Tu Trong Street, Ho Chi Minh City 70000 Vietnam. ${ }^{4}$ Laboratory of Arbovirus, Pasteur Institute in Ho Chi Minh City, 167 Pasteur Street, Ho Chi Minh City 70000, Vietnam. ${ }^{5}$ Department of Immunology \& Microbiology, Pasteur Institute in Ho Chi Minh City, 167 Pasteur Street, Ho Chi Minh City 70000, Vietnam. ${ }^{6}$ University of Medicine and Pharmacy at Ho Chi Minh City, 217 Hong Bang, District 5, Ho Chi Minh City 70000, Vietnam. ${ }^{7}$ Center for Preventive Medicine, 24 Hung Vuong Street, Vinh Long 91000, Vietnam.

Received: 12 July 2013 Accepted: 1 November 2013 Published: 2 December 2013

\section{References}

1. Guzman MG, Halstead SB, Artsob H, Buchy P, Farrar J, Gubler DJ, Hunsperger E, Kroeger A, Margolis HS, Martínez E, Nathan MB, Pelegrino JL, Simmons C, Yoksan S, Peeling RW: Dengue: a continuing global threat. Nat Rev Microbio/ 2010, 8(12 Suppl):S7-S16.

2. World Health Organization (WHO): Epidemiology, burden of disease and transmission. In Dengue: Guidelines for Diagnosis, Treatment, Prevention and Control. Geneva: WHO; 2009:3-21.

3. Kurane I: Dengue hemorrhagic fever with special emphasis on immunopathogenesis. Comp Immunol Microbiol Infect Dis 2007, 30:329-340
4. White NJ: Variation in virulence of dengue virus. Lancet 1999, 354:1401-1402.

5. Halstead SB: Dengue. Lancet 2007, 370:1644-1652.

6. Lan NT, Hirayama K: Host genetic susceptibility to severe dengue infection. Trop Med Health 2011, 39(4 Suppl):73-81.

7. Lan NTP, Kikuchi M, Huong VTQ, Ha DQ, Thuy TT, Tham VD, Tuan HM, Tuong W, Nga CTP, Dat TV, Oyama T, Morita K, Yasunami M, Hirayama K: Protective and enhancing HLA alleles, HLA-DRB1*0901 and HLA$A^{*} 24$, for severe forms of dengue virus infection, dengue hemorrhagic fever and dengue shock syndrome. PLOS Negl Trop Dis 2008, 2:e304.

8. Mongkolsapaya J, Duangchinda T, Dejnirattisai W, Vasanawathana $\mathrm{S}$, Avirutnan P, Jairungsri A, Khemnu N, Tangthawornchaikul N, Chotiyarnwong P, Sae-Jang K, Koch M, Jones Y, McMichael A, Xu X, Malasit P, Screaton G: T cell responses in dengue hemorrhagic fever: Are cross-reactive T cells suboptimal? J Immunol 2006, 176:3821-3829.

9. Chaturvedi UC, Nagar R, Shrivastava R: Macrophage and dengue virus: friend or foe? Indian J Med Res 2006, 124:23-40.

10. Chaturvedi UC, Agarwal R, Elbishbishi EA, Mustafa AS: Cytokine cascade in dengue hemorrhagic fever: implications for pathogenesis. FEMS Immunol Med Microbiol 2000, 28:183-188.

11. Lin CF, Chiu SC, Hsiao YL, Wan SW, Lei HY, Shiau AL, Liu HS, Yeh TM, Chen SH, Liu CC, Lin YS: Expression of cytokine, chemokine, and adhesion molecules during endothelial cell activation induced by antibodies against dengue virus nonstructural protein 1. J Immunol 2005, 174:395-403.

12. Whitehorn J, Simmons CP: The pathogenesis of dengue. Vaccine 2011, 29:7221-7228

13. World Health Organization (WHO): Dengue Haemorrhagic Fever: Diagnosis, Treatment, Prevention and Control. 2nd edition. Geneva: WHO; 1997.

14. World Health Organization (WHO): Dengue: Guidelines for Diagnosis, Treatment, Prevention and Control. Geneva: WHO; 2009.

15. Wilder-Smith A, Ooi EE, Vasudevan SG, Gubler DJ: Update on dengue: epidemiology, virus evolution, antiviral drugs, and vaccine development. Curr Infect Dis Rep 2010, 12:157-164.

16. Kalayanarooj S: Clinical manifestations and management of dengue/DHF/ DSS. Trop Med Health 2011, 39(4 Suppl):83-87.

17. Al-Muhandis N, Hunter PR: The value of educational messages embedded in a community-based approach to combat dengue fever: a systematic review and meta regression analysis. PLoS Negl Trop Dis 2011, 5:e1278.

18. Gibbons RV, Vaughn DW: Dengue: an escalating problem. BMJ 2002 324:1563-1566.

19. Anders KL, Nguyet NM, Chau NV, Hung NT, Thuy T, Lien LB, Farrar J, Wills $B$, Hien TT, Simmons CP: Epidemiological factors associated with dengue shock syndrome and mortality in hospitalized dengue patients in $\mathrm{Ho}$ Chi Minh City, Vietnam. Am J Trop Med Hyg 2011, 84:127-134.

20. Ngo NT, Cao XT, Kneen R, Wills B, Nguyen VM, Nguyen TQ, Chu VT, Nguyen TT, Simpson JA, Solomon T, White NJ, Farrar J: Acute management of dengue shock syndrome: a randomized double-blind comparison of 4 intravenous fluid regimens in the first hour. Clin Infect Dis 2001, 32:204-213.

21. Ha TT, Huy NT, Murao LA, Lan NT, Thuy TT, Tuan HM, Nga CT, Tuong W, Dat TV, Kikuchi M, Yasunami M, Morita K, Huong VT, Hirayama K: Elevated levels of cell-free circulating DNA in patients with acute dengue virus infection. PLoS One 2011, 6:e25969.

22. Furuta T, Murao LA, Lan NT, Huy NT, Huong VT, Thuy $T$, Tham VD, Nga CT, Ha TT, Ohmoto Y, Kikuchi M, Morita K, Yasunami M, Hirayama K, Watanabe $\mathrm{N}$ : Association of mast cell-derived VEGF and proteases in dengue shock syndrome. PLoS Negl Trop Dis 2012, 6:e1505.

23. Wills BA, Oragui EE, Stephens AC, Daramola OA, Dung NM, Loan HT, Chau NV, Chambers M, Stepniewska K, Farrar JJ, Levin M: Coagulation abnormalities in dengue hemorrhagic fever: serial investigations in 167 Vietnamese children with dengue shock syndrome. Clin Infect Dis 2002, 35:277-285

24. Peduzzi $P$, Concato J, Kemper $E$, Holford TR, Feinstein AR: A simulation study of the number of events per variable in logistic regression analysis. J Clin Epidemiol 1996, 49:1373-1379.

25. Harrell FE Jr, Lee KL, Mark DB: Multivariable prognostic models: issues in developing models, evaluating assumptions and adequacy, and measuring and reducing errors. Stat Med 1996, 15:361-387.

26. Little RJA: A test of missing completely at random for multivariate data with missing values. J Am Stat Assoc 1988, 83:1198-1202. 
27. Schafer JL: Multiple imputation: a primer. Stat Methods Med Res 1999, 8:3-15.

28. Kumar CA, Srinivas S: Mining associations in health care data using formal concept analysis and singular value decomposition. J Biol Syst 2010, 18:787-807.

29. Hall M, Frank E, Holmes G, Pfahringer B, Reutemann P, Witten IH: The WEKA data mining software: an update. SIGKDD Explor 2009, 11:10-18.

30. Kohavi R, John GH: Wrappers for feature subset selection. Artif Intell 1997, 97:273-324.

31. Pearl J: Heuristics: Intelligent Search Strategies for Computer Problem Solving. Boston: Addison-Wesley Longman; 1984.

32. Stiell IG, Wells GA: Methodologic standards for the development of clinical decision rules in emergency medicine. Ann Emerg Med 1999, 33:437-447.

33. Huang J, Ling CX: Using AUC and accuracy in evaluating learning algorithms. IEEE Trans Knowl Data Eng 2005, 17:299-310.

34. Kohavi R: A study of cross-validation and bootstrap for accuracy estimation and model selection. In Proceedings of the 14th International Joint Conference on Artificial Intelligence, Volume 2. San Francisco: Morgan Kaufmann; IJCAI (U S); 1995:1137-1143.

35. Witten $\mid H$, Frank E: Data Mining: Practical Machine Learning Tools and Techniques. 2nd edition. San Francisco: Morgan Kaufmann; 2005.

36. Nadeau C, Bengio Y: Inference for the generalization error. Mach Learn 2003, 52:239-281.

37. Kerr WT, Lau EP, Owens GE, Trefler A: The future of medical diagnostics: large digitized databases. Yale J Biol Med 2012, 85:363-377.

38. Li L, Tang H, Wu Z, Gong J, Gruidl M, Zou J, Tockman M, Clark RA: Data mining techniques for cancer detection using serum proteomic profiling. Artif Intell Med 2004, 32:71-83.

39. Denekamp Y: Clinical decision support systems for addressing information needs of physicians. Isr Med Assoc J 2007, 9:771-776.

40. Kong L, Milbrandt EB, Weissfeld LA: Advances in statistical methodology and their application in critical care. Curr Opin Crit Care 2004, 10:391-394.

41. Moser SA, Jones WT, Brossette SE: Application of data mining to intensive care unit microbiologic data. Emerg Infect Dis 1999, 5:454-457.

42. Potts JA, Gibbons RV, Rothman AL, Srikiatkhachorn A, Thomas SJ, Supradish PO, Lemon SC, Libraty DH, Green S, Kalayanarooj S: Prediction of dengue disease severity among pediatric Thai patients using early clinical laboratory indicators. PLoS Negl Trop Dis 2010, 4:e769.

43. Dal Moro F, Abate A, Lanckriet GR, Arandjelovic G, Gasparella P, Bassi P, Mancini M, Pagano F: A novel approach for accurate prediction of spontaneous passage of ureteral stones: support vector machines. Kidney Int 2006, 69:157-160

44. Anderson B, Hardin JM, Alexander DD, Grizzle WE, Meleth S, Manne U: Comparison of the predictive qualities of three prognostic models of colorectal cancer. Front Biosci (Elite Ed) 2010, 2:849-856.

45. Wang CJ, Li YQ, Wang L, Li LL, Guo YR, Zhang LY, Zhang MX, Bie RH: Development and evaluation of a simple and effective prediction approach for identifying those at high risk of dyslipidemia in rural adult residents. PLoS One 2012, 7:e43834

46. Takayama T, Ebinuma H, Tada S, Yamagishi Y, Wakabayashi K, Ojiro K, Kanai T, Saito H, Hibi T, Keio Association for the Study of Liver Diseases: Prediction of effect of pegylated interferon $a-2 b$ plus ribavirin combination therapy in patients with chronic hepatitis $\mathrm{C}$ infection. PLoS One 2011, 6:e27223.

47. Eller-Vainicher C, Chiodini I, Santi I, Massarotti M, Pietrogrande L, Cairoli E, Beck-Peccoz P, Longhi M, Galmarini V, Gandolini G, Bevilacqua M, Grossi E: Recognition of morphometric vertebral fractures by artificial neural networks: analysis from GISMO Lombardia Database. PLoS One 2011, 6:e27277.

48. Austin PC: A comparison of regression trees, logistic regression, generalized additive models, and multivariate adaptive regression splines for predicting AMI mortality. Stat Med 2007, 26:2937-2957.

49. Jonsdottir T, Hvannberg ET, Sigurdsson H, Sigurdsson S: The feasibility of constructing a Predictive Outcome Model for breast cancer using the tools of data mining. Expert Syst App/ 2008, 34:108-118.
50. Buri L, Hassan C, Bersani G, Anti M, Bianco MA, Cipolletta L, Di Giulio E, Di Matteo G, Familiari L, Ficano L, Loriga P, Morini S, Pietropaolo V, Zambelli A, Grossi E, Intraligi M, Buscema M, SIED Appropriateness Working Group: Appropriateness guidelines and predictive rules to select patients for upper endoscopy: a nationwide multicenter study. Am J Gastroenterol 2010, 105:1327-1337.

51. Hosmer DW Jr, Lemeshow S: Assessing the fit of the model. In Applied Logistic Regression. 2nd edition. New York: John Wiley \& Sons; 2000:143-202.

doi:10.1186/cc13135

Cite this article as: Huy et al:: Development of clinical decision rules to predict recurrent shock in dengue. Critical Care 2013 17:R280

\section{Submit your next manuscript to BioMed Central and take full advantage of:}

- Convenient online submission

- Thorough peer review

- No space constraints or color figure charges

- Immediate publication on acceptance

- Inclusion in PubMed, CAS, Scopus and Google Scholar

- Research which is freely available for redistribution

Submit your manuscript at www.biomedcentral.com/submit
C) BioMed Central 